\title{
Is Biologic Therapy in Inflammatory Bowel Disease Contributing to the Obesity Epidemic? Just Weight One Year
}

\author{
Aditi Mulgund $^{1} \cdot$ Daniel Stein ${ }^{1}$
}

Published online: 24 August 2020

(c) Springer Science+Business Media, LLC, part of Springer Nature 2020

\begin{abstract}
Although a comment about others' weight may get some people slapped in the face, frequently in the inflammatory bowel disease (IBD) population, weight gain is regarded as a positive indicator of disease control. Nevertheless, obesity has become a very serious problem in the USA and worldwide [1]. Since IBD patients have not been left out of the obesity epidemic, IBD clinicians need to ensure that they do not worsen the problem.
\end{abstract}

Obesity in patients with IBD is rising, with cross-sectional studies showing that between 15 and $40 \%$ of patients with IBD are obese, although the pathogenesis is not entirely understood [2-4]. Smoking cessation and the use of corticosteroids for flares can contribute to weight gain. Obesity in IBD may result in suboptimal response to therapy and can heighten the difficulty of colorectal surgery by increasing the risk of perioperative complications and by impairing ostomy function.

In this issue of Digestive Diseases and Sciences, Borren et al. evaluated a prospective cohort of 269 patients after initiating biologic therapy while monitoring weight increase from baseline [5]. These patients were receiving biologic therapy with either infliximab, adalimumab, vedolizumab, or ustekinumab, and had either Crohn's disease (CD) or ulcerative colitis (UC). Study assessments were performed at weeks $0,14,30$, and 54 ; the primary outcome was defined as the difference between baseline weight and that at each of the prespecified weeks. The authors also collected data to reflect disease activity, including the Harvey-Bradshaw Index (HBI) for Crohn's disease and the Simple Clinical Colitis Activity Index (SCCAI) for UC as well as quality-of-life

Daniel Stein

dstein@mcw.edu

Aditi Mulgund

amulgund@mcw.edu

1 Division of Gastroenterology and Hepatology, Medical College of Wisconsin, 9200 W. Wisconsin Ave., Milwaukee, WI 53226, USA metrics with the short inflammatory bowel disease questionnaire (SIBDQ). The authors ultimately found that from baseline, weight increased by a mean of $1.29 \mathrm{~kg}$ by week 54 . This weight gain was particularly significant by weeks 30 and 54 in patients receiving anti-TNF therapy, by week 30 in patients receiving vedolizumab, and at all three timepoints in patients receiving ustekinumab. The authors further compared the association between clinical disease activity and weight gain, finding no correlation between disease activity at baseline and weight gain at the time points studied. The SIBDQ score did not differ across therapeutic classes but was significantly improved by week 14 and continued to improve until the end of the study. There was no association found between the SIBDQ score and the increase in weight at the different time points, suggesting that improvement in clinical disease activity and patient assessment of their disease was not in itself the reason for weight gain [5].

The authors recruited a large cohort of patients, adjusted for age, gender, disease type, C-reactive protein (CRP), smoking history, therapy, and disease activity score using a prospective and observational study design. The study looks at multiple classes of biologic agents, whereas previous data have concentrated more deeply on anti-TNF agents [2]. Nevertheless, weights were taken at different times of the day and can vary drastically throughout the day. The authors also excluded patients with $a>10 \mathrm{~kg}$ weight gain, as these patients' weights may have been inaccurate. This could have skewed the patient cohort results toward a more modest weight gain and might have resulted in a more significant weight gain in a portion of patients.

Weight gain in inflammatory bowel disease is a real concern. Though many patients with active IBD may be underweight and malnourished, most of the patients included in this study had a normal BMI or were already overweight or obese. The current literature suggests that patients who are overweight or obese are likely to have more frequent clinic visits, hospitalizations, disease flares, and require escalation of therapy [2]. Yet, not all studies portend an inferior 
outcome in patients with IBD who are obese or overweight $[4,6]$. A large cross-sectional study noted no negative longterm outcomes in patients with Crohn's disease and obesity, and another study demonstrated no increased health utilization among these patients $[4,6]$.

Borren et al. add to a growing area of research on obesity, particularly within IBD patients, in a large prospective study. Anti-TNF agents, vedolizumab, and ustekinumab all have been proven efficacious in randomized controlled trials advocating for their use over placebo in the treatment of UC and CD [7, 8]. These medications may have some negative aspects, such as their cost, infection risk, or, as this study suggests, weight gain. Nonetheless, this is an era in which effective therapy for treating IBD exists and the monetary and non-monetary costs of longstanding untreated inflammatory bowel disease are much greater than the risks of a modest weight gain. This study demonstrates that anti-TNF agents, vedolizumab, and ustekinumab were all associated with some amount of weight gain, though modest and comparable across all classes of medications, with most patients included gaining up to $2 \mathrm{~kg}$ of weight over the course of 1 year. Although clinicians need to ultimately utilize shared decision making with their patients, some weight gain may occur, though it remains modest considering the benefits of biologic therapy for patients with $\mathrm{CD}$ and $\mathrm{UC}$, outweighing the risk of untreated disease.

\section{Compliance with Ethical Standards}

Conflict of interest The authors have nothing to disclose.

\section{References}

1. Blüher M. Obesity: global epidemiology and pathogenesis. Nat Rev Endocrinol. 2019;15:288-298. https://doi.org/10.1038/s4157 4-019-0176-8.
2. Pavelock N, Masood U, Minchenberg S, Heisig D. Effects of obesity on the course of inflammatory bowel disease. Proc Bayl Univ Med Cent. 2019;32:14-17. https://doi.org/10.1080/08998 280.2018.1542887.

3. Pringle PL, Stewart KO, Peloquin JM, et al. Body mass index, genetic susceptibility, and risk of complications among individuals with Crohn's disease. Inflamm Bowel Dis. 2015;21:2304-2310. https://doi.org/10.1097/mib.0000000000000498.

4. Nic Suibhne T, Raftery TC, McMahon O, Walsh C, O'Morain C, O'Sullivan M. High prevalence of overweight and obesity in adults with crohn's disease: associations with disease and lifestyle factors. J Crohns Colitis. 2013;7:e241-e248.

5. Borren NK, Tan W, Jess AT, Li PM, et al. Assessment of body weight changes in patients with inflammatory bowel diseases initiating biologic therapy: a prospective cohort study. Dig Dig Sci. (Epub ahead of print). https://doi.org/10.1007/s10620-020-06442 $-9$.

6. Seminerio JL, Koutroubakis IE, Ramos-Rivers C, et al. Impact of obesity on the management and clinical course of patients with inflammatory bowel disease. Inflamm Bowel Dis. 2015;21:28572863. https://doi.org/10.1097/mib.0000000000000560.

7. Feuerstein JD, Isaacs KL, Schneider Y, et al. AGA clinical practice guidelines on the management of moderate to severe ulcerative colitis. Gastroenterology. 2020;158:1450-1461.

8. Terdiman JP, Gruss CB, Heidelbaugh JJ, Sultan S, Falck-Ytter YT. AGA Institute Clinical Practice and Quality Management Committee. American gastroenterological association institute guideline on the use of thiopurines, methotrexate, and antiTNF- $\alpha$ biologic drugs for the induction and maintenance of remission in inflammatory Crohn's disease. Gastroenterology. 2013;145:1459-1463.

Publisher's Note Springer Nature remains neutral with regard to jurisdictional claims in published maps and institutional affiliations. 